\title{
CONSTITUINTES QUÍMICOS E ATIVIDADE ANTIOXIDANTE IN VIVO DE FLAVONOIDES ISOLADOS DE Clusia lanceolata (Clusiaceae) $)^{\#}$
}

Rafaela O. Ferreira ${ }^{a} *$, Almir R. de Carvalho Junior ${ }^{\mathrm{a}}$, Cristiano J. Riger ${ }^{\mathrm{a}}$, Rosane N. Castro ${ }^{\mathrm{a}}$, Tania M. S. da Silva ${ }^{\mathrm{b}}$ e Mário G. de Carvalho ${ }^{\mathrm{a}}$

${ }^{a}$ Departamento de Química, Instituto de Ciências Exatas, Universidade Federal Rural do Rio de Janeiro, 23851-970, Seropédica

- RJ, Brasil

${ }^{\mathrm{b}}$ Departamento de Ciências Moleculares, Universidade Federal Rural de Pernambuco, 52171-900, Recife - PE, Brasil

Recebido em 14/03/2016; aceito em 19/05/2016; publicado na web em 20/07/2016

\begin{abstract}
CHEMICAL CONSTITUENTS AND IN VIVO ANTIOXIDANT ACTIVITY OF FLAVONOIDS ISOLATED OF Clusia lanceolata (Clusiaceae). The phytochemical investigation of Clusia lanceolata leaves yielded fourteen compounds including six flavones, vitexin, isovitexin, isovitexin-2"- $O-\alpha-L$-rhamnopyranoside, vitexin-2"- $O-\alpha-L$-rhamnopyranoside, orientin and isoorientin, two phaeophytins [132-hydroxy-(132-S-)-phaeophytin a and $13^{2}$-hydroxy-(132-R)-phaeophytin a], two triterpenes, $\alpha$ - and $\beta$-amyrin, and four steroids, $\beta$-sitosterol, stigmasterol, sitosterone and stigmasterone. Their structures were assigned based on spectroscopic analysis, including two-dimensional NMR techniques and comparison with literature data. The antioxidant capacity in vivo of vitexin and mixing isovitexin-2"-O- $\alpha$ - $L$-rhamnopyranoside and vitexin-2"-O- $\alpha-L$-rhamnopyranoside was evaluated using the yeast Saccharomyces cerevisiae as the biological system model and hydrogen peroxide as a stressor agent. The results showed that the tested flavonoids were able to protect the yeast cell against the oxidative damage caused by $\mathrm{H}_{2} \mathrm{O}_{2}$.
\end{abstract}

Keywords: Clusiaceae; Clusia lanceolata; flavonoids; antioxidants; Saccharomyces cerevisiae.

\section{INTRODUÇÃo}

Plantas da família Clusiaceae têm sido motivo de estudos fitoquímicos, por apresentarem metabólitos especiais com estruturas diversificadas e com atividades biológicas, principalmente os pertencentes às classes de benzofenonas, biflavonoides e xantonas. ${ }^{1,2}$ Nesta família, o gênero Clusia, constituído por aproximadamente 250 espécies, ${ }^{3}$ tem recebido mais atenção quanto ao estudo químico e farmacológico. Estudos realizados com este grupo de plantas levaram a caracterização de metabólitos especiais pertencentes a várias classes, tais como: benzofenonas, floroglucinóis, biflavonoides, bifenilas e xantonas. ${ }^{1,4-7}$ Entre as atividades relatadas para substâncias e/ou extratos obtidos de plantas deste gênero, destacam-se as propriedades anti-inflamatórias, antimicrobianas e antioxidantes..$^{5,6,8}$

Clusia lanceolata Cambess, popularmente conhecida como cebola-da-mata, é uma espécie endêmica nas restingas fluminenses, cujos extratos de folhas apresentaram atividade antioxidante in vitro. ${ }^{9}$ Não há registro do uso medicinal e poucos são os dados sobre estudo fitoquímico relacionado a esta espécie. Em trabalho anterior descrevemos a identificação de isovitexina-2"- $O-\alpha-L$ ramnopiranosídeo, vitexina-2"'-O- $\alpha$ - $L$-ramnopiranosídeo, isoorientina e orientina em extratos de folhas de $C$. lanceolata por CLAEEM. ${ }^{9}$ Em continuação a busca por substâncias com propriedades antioxidantes em espécies do gênero Clusia,${ }^{5,9}$ são apresentados neste trabalho os resultados do estudo fitoquímico de extratos de folhas de $C$. lanceolata e avaliação da atividade antioxidante in vivo de algumas das substâncias isoladas.

*e-mail: rafaellaoliveira@ufrrj.br \#This paper is part of the PubliSBQ Special Issue in honor of the late Prof. Angelo da Cunha Pinto.

\section{PARTE EXPERIMENTAL}

\section{Procedimentos experimentais gerais}

Para cromatografia em camada delgada (CCD) utilizaram-se placas de sílica gel $\mathrm{PF}_{254}$ (Whatman e Silicle), as quais foram observadas sob luz UV 254 e $366 \mathrm{~nm}$ e reveladas com soluções de sulfato cérico e vanilina sulfúrica, seguida de aquecimento. As separações cromatográficas em coluna (CC) foram realizadas utilizando-se gel de sílica 60 (Vetec, 70-230 mesh e 230-400 mesh) e Sephadex LH20 (Sigma-Aldrich). Os experimentos de $\mathrm{RMN}$ de ${ }^{1} \mathrm{H}$ e ${ }^{13} \mathrm{C}$ (uni- e bidimensionais) foram obtidos em espectrômetro Bruker AVANCE II $(500 / 125 \mathrm{MHz})$, utilizando-se $\mathrm{CDCl}_{3}, \mathrm{DMSO}-d_{6}$ e $\mathrm{CD}_{3} \mathrm{OD}$ como solventes e os sinais residuais destes em relação ao TMS como referência. Os espectros no IV foram registrados em espectrômetro VERTEX-70, tendo sido as amostras preparadas sob a forma de filme sobre cela de $\mathrm{NaCl}$ ou pastilha de $\mathrm{KBr}$. Os espectros de massas de baixa resolução foram obtidos no espectrômetro GCMS-QP2010 Plus, Shimadzu. As análises fotométricas foram registradas em espectrofotômetro UV-VIS da marca Shimadzu, modelo UV-mini 1240.

\section{Coleta e identificação do material botânico}

O material botânico, folhas de Clusia lanceolata, foi coletado na Restinga de Grumari no munícipio do Rio de Janeiro-RJ em abril de 2012. O material botânico foi identificado pelo técnico Me. Thiago Amorim (RBR - UFRRJ). A exsicata de número 35424 está depositada no herbário RBR, da UFRRJ.

\section{Extração e isolamento dos constituintes químicos}

Folhas secas $(230,0 \mathrm{~g})$ foram trituradas e submetidas à extração com $\mathrm{CH}_{2} \mathrm{Cl}_{2}$ e, posteriormente, em $\mathrm{MeOH}$, através de maceração a temperatura ambiente. As soluções resultantes após filtração foram concentradas sob pressão reduzida, fornecendo os extratos brutos em 
$\mathrm{CH}_{2} \mathrm{Cl}_{2}$ (CLFD, 23,0 g) e $\mathrm{MeOH}$ (CLFM, 20,0 g). Parte do extrato CLFM (17,0 g) foi solubilizada em uma solução $\mathrm{MeOH} / \mathrm{H}_{2} \mathrm{O}(7: 3)$ e submetido a partições líquido/líquido, e foram obtidas as frações em n-hexano (CLFMH, 1,6 g), $\mathrm{CHCl}_{3}$ (CLFMC, 1,5 g), AcOEt (CLFMAc, $3,5 \mathrm{~g})$ e $\mathrm{BuOH}$ (CLFMBu, 2,0 g), respectivamente. A fração AcOEt forneceu um precipitado identificado como vitexina (1, 110,0 mg).

Parte da fração AcOEt (3,0 g) foi filtrada em Sephadex LH-20 usando $\mathrm{MeOH}$ como eluente, foram obtidas 6 frações, após monitoramento por CCD. A quinta fração forneceu a isovitexina (2, 5,0 mg). A segunda e a quarta frações foram filtradas novamente em Sephadex LH-20 usando $\mathrm{MeOH}$ como eluente e forneceram, respectivamente, a mistura de isovitexina-2"- $O$ - $\alpha-L$-ramnopiranosídeo e vitexina-2"- $O$ $\alpha$ - $L$-ramnopiranosídeo $(3+4,40,0 \mathrm{mg})$ e a mistura de isoorientina, orientina e isovitexina $(5+6+2,12,0 \mathrm{mg})$.

O extrato CLFD (20,0 g) foi fracionado através de cromatografia em coluna (CC) em gel de sílica 60, empregando-se como eluentes, em modo isocrático, $n$-hexano, $\mathrm{CH}_{2} \mathrm{Cl}_{2}$, AcOEt e $\mathrm{MeOH}$, fornecendo quatro frações. A segunda fração $(7,0 \mathrm{~g})$ foi submetida a $\mathrm{CC}$ em sílica gel 60, empregando-se como eluentes $n$-hexano, $\mathrm{CH}_{2} \mathrm{Cl}_{2}$ e AcOEt em gradiente crescente de polaridade, fornecendo as misturas de $\alpha$ e $\beta$-amirina $(7+8,1,2 \mathrm{~g})$ e $\beta$-sitosterol com estigmasterol $(9+10$, $30,0 \mathrm{mg}$ ). A terceira fração $(10,0 \mathrm{~g})$ foi submetida a CC (sílica gel 60) e eluída com $\mathrm{CH}_{2} \mathrm{Cl}_{2}$, AcOEt e $\mathrm{MeOH}$ em gradiente crescente de polaridade, fornecendo 10 frações. A terceira fração desta coluna forneceu a mistura de rel-132-hidroxi-(132-S-)-feofitina a e rel-132hidroxi-(132-R)-feofitina a $(11+12,70,0 \mathrm{mg})$. A quinta fração foi submetida à nova $\mathrm{CC}$ (sílica gel 60) e eluída com $\mathrm{CH}_{2} \mathrm{Cl}_{2}$ e AcOEt, fornecendo a mistura de sitosterona e estigmasterona $(13+14,35,0$ mg). Uma solução de $1,0 \mathrm{mg} / \mathrm{mL}$ em metanol grau HPLC da mistura de isovitexina-2"- $O$ - $\alpha$ - $L$-ramnopiranosídeo (3) e vitexina-2"'-O$\alpha$ - $L$-ramnopiranosídeo (4) foi injetada no aparelho CLAE-DAD da Shimadzu com coluna C18 Betasil-Thermo $(25 \mathrm{~cm} \mathrm{x} \mathrm{4,6} \mathrm{mm,} 5 \mu \mathrm{m})$ a $29{ }^{\circ} \mathrm{C}$. As substâncias foram eluídas com gradiente de fase móvel binária composta por: (A) ácido acético a 1\% em água e (B) metanol. O sistema de eluição teve como condição inicial a eluição de $60 \%$ de B por 10 minutos, seguido de um gradiente linear de B até $28 \%$ até 12 minutos e, $28 \%$ de B até 15 minutos, com fluxo de $1,3 \mathrm{~mL} / \mathrm{min}$ e volume de injeção de $20 \mu \mathrm{L}$ das substâncias. A faixa de comprimento de onda ( $\lambda) 200 \mathrm{~nm}$ a $500 \mathrm{~nm}$ foi utilizada na investigação. Todas as substâncias foram submetidas à análise de ressonância magnética nuclear de ${ }^{1} \mathrm{H}$ e ${ }^{13} \mathrm{C}$ e, adicionalmente, as substâncias 7, 8, 13 e 14 foram identificadas inclusive com análise por CG-EM.

Vitexina (1): Sólido amarelo. RMN ${ }^{1} \mathrm{H}\left(\mathrm{DMSO}-d_{6}, 500 \mathrm{MHz}\right) \delta_{\mathrm{H}}$ (mult.; $J$ em Hz; H): 6,79 ( $s$; H-3), 13,17 ( $s$; OH-5), 6,27 ( $s$; H-6), $8,02\left(d ; J=8,5 ; \mathrm{H}^{\prime} 2^{\prime}, 6^{\prime}\right), 6,87(d ; J=8,5 ; \mathrm{H}-3$ ', 5'), $4,73(d ; J=$ 9,8; H-1"). RMN ${ }^{13} \mathrm{C}$ (DMSO- $\left.d_{6}, 125 \mathrm{MHz}\right) \delta_{\mathrm{C}}: 163,9$ (C-2), 102,5 (C-3), 182,1 (C-4), 160,4 (C-5), 98,1 (C-6), 162,6 (C-7), 104,6 (C8), 156,0 (C-9), 104,0 (C-10), 121,6 (C-1'), 129,0 (C-2',6'), 115,8 (C-3',5'), 73,4 (C-1').

Isovitexina (2): Sólido amarelo. $\mathrm{RMN}{ }^{1} \mathrm{H}\left(\mathrm{DMSO}-d_{6}, 500 \mathrm{MHz}\right)$ $\delta_{\mathrm{H}}$ (mult.; $J$ em Hz; H): 6,66 ( $s$; H-3), 13,53 ( $s$; OH-5), 6,32 ( $s$; H-6), $7,88(d ; J=8,5$; H-2', 6'), 6,90 ( $d ; J=8,5 ; \mathrm{H}-3$ ', 5'), 4,57 ( $d ; J=$ 10,$0 ; \mathrm{H}-1$ "). RMN ${ }^{13} \mathrm{C}$ (DMSO- $\left.d_{6}, 125 \mathrm{MHz}\right) \delta_{\mathrm{C}}: 163,9$ (C-2), 103,0 (C-3), 182,2 (C-4), 161,4 (C-5), 109,0 (C-6), 163,7 (C-7), 94,0 (C8), 156,6 (C-9), 103,6 (C-10), 121,4 (C-1'), 128,7 (C-2',6'), 116,3 (C-3',5'), 73,3 (C-1').

Isovitexina-2”-O- $\alpha$-L-ramnopiranosídeo (3): Sólido amarelo. $\mathrm{RMN}{ }^{1} \mathrm{H}\left(\mathrm{DMSO}-d_{6}, 500 \mathrm{MHz}\right)$ e ${ }^{13} \mathrm{C}$ (DMSO- $\left.d_{6}, 125 \mathrm{MHz}\right)$. Ver Tabela 1.

Vitexina-2"-O- $\alpha$-L-ramnopiranosídeo (4): Sólido amarelo. RMN ${ }^{1} \mathrm{H}$ (DMSO- $d_{6}, 500 \mathrm{MHz}$ ) e ${ }^{13} \mathrm{C}$ (DMSO- $d_{6}, 125 \mathrm{MHz}$ ). Ver Tabela 1. Isoorientina (5): Sólido amarelo. RMN ${ }^{1} \mathrm{H}\left(\mathrm{DMSO}-d_{6}, 500 \mathrm{MHz}\right)$ $\delta_{\mathrm{H}}$ (mult.; $J$ em Hz; H): 6,67 ( $\left.s ; \mathrm{H}-3\right), 13,57$ ( $s$ OH-5), 6,47 ( $s ; \mathrm{H}-6$ ), $7,40(d ; J=2,5 ; \mathrm{H}-2$ ') $, 6,88$ ( $d ; J=9,0 ; \mathrm{H}-5$ '), 7,42 (dd; $J=2,5$ e 9,0; H-6'), 4,58 ( $d$; $J=10,0$; H-1"). RMN ${ }^{13} \mathrm{C}$ (DMSO- $\left.d_{6}, 125 \mathrm{MHz}\right) \delta_{\mathrm{C}}$ : 163,6 (C-2), 102,8 (C-3), 181,9 (C-4), 160,7 (C-5), 108,9 (C-6), 163,6 (C-7), 93,5 (C-8), 156,2 (C-9), 103,3 (C-10), 121,3 (C-1'), 113,3 (C-2'), 145,8 (C-3'), 149,8 (C-4'), 116,1 (C-5'), 119,0 (C-6'), 73,1 (C-1').

Orientina (6): Sólido amarelo. RMN ${ }^{1} \mathrm{H}\left(\mathrm{DMSO}-d_{6}, 500 \mathrm{MHz}\right)$ $\delta_{\mathrm{H}}$ (mult.; $J$ em Hz; H): 6,64 ( $\left.s ; \mathrm{H}-3\right), 13,18$ ( $\left.s ; \mathrm{OH}-5\right), 6,26$ ( $s$; H-6), 7,48 ( $s l$; H-2'), 6,92 ( $d ; J=8,0 ; \mathrm{H}-5$ '), 7,53 ( $d d ; J=2,0$ e 8,0; H-6'), $4,68(d ; J=9,0 ; \mathrm{H}-1 ")$.

Mistura de $\alpha$-amirina (7) e $\beta$-amirina (8): Cristais brancos. RMN ${ }^{1} \mathrm{H} \mathrm{e}{ }^{13} \mathrm{C}$ e massas em acordo com dados da literatura. ${ }^{10}$

Mistura de $\beta$-sitosterol (9) e estigmasterol (10): Cristais brancos. RMN ${ }^{1} \mathrm{H}$ e ${ }^{13} \mathrm{C}$ em acordo com dados da literatura. ${ }^{11}$

Mistura de 132-hidroxi-(132-S-)-feofitina (11) e 132-hidroxi-(132$R$ )-feofitina (12): Sólido verde. UV, IV, $\mathrm{RMN}{ }^{1} \mathrm{H}$ e ${ }^{13} \mathrm{C}$ em acordo com dados da literatura. ${ }^{12}$

Mistura de sitosterona (13) e estigmasterona (14): Sólido amarelo. $\mathrm{RMN}{ }^{1} \mathrm{H}$ e ${ }^{13} \mathrm{C}$ e massas em acordo com dados da literatura. ${ }^{13}$

\section{Determinação da atividade antioxidante in vivo}

\section{Linhagens de Saccharomyces cerevisiae e condições de crescimento}

A cepa BY4741 (MATa; his3; leu2; met15; ura3) foi adquirida da empresa Euroscarf (Frankfurt, Alemanha). Seus estoques foram mantidos em meio de cultura sólido YPD 2\% (1\% de extrato de levedura, $2 \%$ de glicose, $2 \%$ de peptona e $2 \%$ de ágar). Para todos os experimentos, as células foram cultivadas em meio YPD $2 \%$ líquido usando um agitador orbital a $28^{\circ} \mathrm{C}$ e $160 \mathrm{rpm}$ com a relação de volume/meio no frasco de 5:1. Componentes do meio de cultura foram adquiridos da Difco. A concentração celular foi determinada medindo a absorbância a $570 \mathrm{~nm}$. O fator de conversão da absorbância em peso seco foi calculado por meio de filtração de $10 \mathrm{~mL}$ da suspensão de células através de um filtro Milipore $(0,45 \mu \mathrm{m})$ e desidratado a $80^{\circ} \mathrm{C}$ até peso constante.

\section{Estresse oxidativo, viabilidade celular e disfunção mitocondrial}

As células de levedura foram recolhidas na $1^{\text {a }}$ fase exponencial de crescimento ( $1,0 \mathrm{mg}$ de peso seco/mL), e em seguida incubadas com as substâncias na concentração de $25 \mu \mathrm{g} / \mathrm{mL}$ durante $120 \mathrm{~min}$ a $28^{\circ} \mathrm{C}$ e $160 \mathrm{rpm}$. As células foram recolhidas por centrifugação e lavadas duas vezes com tampão fosfato $50 \mathrm{mM}, \mathrm{pH}$ 6,0. Em seguida, as células livres do meio foram ressuspensas no mesmo tampão fosfato contendo $\mathrm{H}_{2} \mathrm{O}_{2}$ na concentração de $1,0 \mathrm{mM}$, e mantidas por $60 \mathrm{~min}$ a $28^{\circ} \mathrm{C}$ e $160 \mathrm{rpm}$. A viabilidade celular foi determinada por plaqueamento em meio YPD sólido, após diluição apropriada, antes e depois das condições de estresse oxidativo, e foi expressa como percentagem de sobrevivência. As placas foram incubadas a $28{ }^{\circ} \mathrm{C}$ durante 72 horas e as colônias contadas. Já para detectar a disfunção mitocondrial as células passaram pelo mesmo tratamento anterior, porém o plaqueamento foi realizado em meio sólido YPGly 4\% (1\% de extrato de levedura, 4\% de glicerol, $2 \%$ de peptona e $2 \%$ de ágar), usado para testar a incapacidade das células de crescer sob meio estritamente aeróbio (glicerol). A viabilidade foi medida como percentagem de células viáveis, cultivadas em YPD 2\% que sobreviveram após incubação com vitexina (1) e a mistura de isovitexina-2"- $O-\alpha-L$ ramnopiranosídeo (3) e vitexina-2"- $O$ - $\alpha$ - $L$-ramnopiranosídeo (4) após o estresse com peróxido de hidrogênio. Já a taxa de danos nas mitocôndrias foi determinada como a diferença entre o número de colônias contadas em placas YPGly 4\% e YPD 2\% após incubação com as substâncias (1 e $3+4$ ) após o estresse com peróxido de hidrogênio. ${ }^{14}$

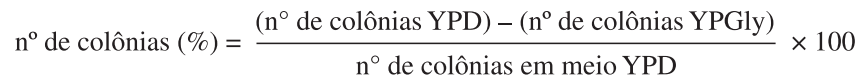


Todos os experimentos foram realizados com três repetições. A solução de $\mathrm{H}_{2} \mathrm{O}_{2}(30 \%)$ foi obtida da empresa Vetec (Rio de Janeiro, Brasil).

\section{Análise estatística}

Os resultados foram submetidos a tratamento estatístico através do teste de Tuckey, utilizando-se o programa GraphPad Prism 5.0 DEMO.

\section{RESULTADOS E DISCUSSÃO}

O fracionamento cromatográfico de extratos de folhas de Clusia lanceolata conduziu ao isolamento da vitexina (1), isovitexina (2), isovitexina-2"- $O$ - $\alpha$ - $L$-ramnopiranosídeo (3), vitexina-2"- $O-\alpha-L$ ramnopiranosídeo (4), isoorientina (5), orientina (6), $\alpha$ e $\beta$-amirina (7 e 8), $\beta$-sitosterol (9), estigmasterol (10), rel-132-hidroxi-(132-S-)feofitina a (11), rel-132-hidroxi-(132-R)-feofitina a (12), sitosterona (13) e estigmasterona (14) (Figura 1). A identificação das substâncias baseou-se na análise de espectros e comparação de dados com valores descritos na literatura. ${ }^{10-13,15-19}$

As substâncias 1, 2, 5 e $\mathbf{6}$ foram identificadas como flavonas, através da análise dos dados de $\mathrm{RMN}{ }^{1} \mathrm{He}$ e ${ }^{13} \mathrm{C}$ (ver parte experimental) e espectros bidimensionais $\operatorname{COSY}^{1} \mathrm{H}-{ }^{1} \mathrm{H}, \mathrm{HMQC}$ e HMBC, além da comparação com dados publicados anteriormente. Nos espectros de RMN ${ }^{1} \mathrm{H}$ as flavonas mostram um singleto entre $\delta_{\mathrm{H}} 6,0$ e 8,0 característico de hidrogênio ligado ao C-3, sendo que no espectro de RMN ${ }^{13} \mathrm{C}$ o sinal correspondente a este carbono mostra sinal em torno de $\delta_{\mathrm{C}}$
102. A substância 1 foi identificada como vitexina, ${ }^{15}$ e as substâncias 2,5 e 6 foram identificadas como isovitexina, isoorientina e orientina, respectivamente. ${ }^{15,16,17}$

A análise por CLAE-DAD da fração contendo as substâncias 3 e $\mathbf{4}$ mostrou a presença de dois picos com espectros de UV exibindo duas bandas com máximos de absorção em 270 e 350, característicos de núcleos de flavonas. $\mathrm{O}$ espectro de $\mathrm{RMN}{ }^{1} \mathrm{H}$ de 3 apresentou sinais característicos de apigenina 6- $C$-substituída, sendo caracterizado pela presença de dois dupletos em $\delta_{\mathrm{H}} 7,92\left(J=8,5 \mathrm{~Hz} ; \mathrm{H}-2^{\prime} / 6^{\prime}\right)$ e $\delta_{\mathrm{H}}$ $6,93\left(J=8,5 \mathrm{~Hz} ; \mathrm{H}_{-} 3^{\prime} / 5^{\prime}\right)$, sugerindo um sistema do tipo AA'XX', dois singletos em $\delta_{\mathrm{H}} 6,77(\mathrm{H}-3)$ e $\delta_{\mathrm{H}} 6,54(\mathrm{H}-8)$, e ausência de sinal característico de H-6. Adicionalmente, observou-se sinais em $\delta_{\mathrm{H}}$ 4,66 (H-1") e $\delta_{\mathrm{H}} 5,08$ (H-1"') que permitiram atribuir a presença de duas unidades glicosídicas. Os espectros de RMN ${ }^{13} \mathrm{C}$ e HMQC de $\mathbf{3}$ corroboraram com a sugestão de duas unidades de carboidrato pela presença de sinais para dois carbonos anoméricos em $\delta_{\mathrm{C}} 71,6$ (C-1") e $\delta_{\mathrm{C}} 104,4$ (C-1"'), além dos sinais para um carbono metilênico em $\delta_{\mathrm{C}} 61,8$ (C-6") e carbono metílico em $\delta_{\mathrm{C}} 17,6$ (C-6"”). Após comparação com dados da literatura ${ }^{18}$ propôs-se a presença de uma unidade de glicose e uma de ramnose. As correlações ${ }^{3} J_{\mathrm{HC}}$ observadas no HMBC entre os picos em $\delta$ 4,66/109,0 (H-1"/C-6) e $\delta 5,08 / 74,6$ (H-1"'/C-2") confirmaram a conectividade do dissacarídeo na molécula. Por conseguinte, a análise detalhada dos dados de RMN de ${ }^{1} \mathrm{H}$ e ${ }^{13} \mathrm{C}, \operatorname{COSY}{ }^{1} \mathrm{H}-{ }^{1} \mathrm{H}, \mathrm{HMQC}$ e HMBC e a comparação com dados descritos na literatura permitiram identificar a substância $\mathbf{3}$ como isovitexina-2"-O- $\alpha$-L-ramnopiranosídeo ${ }^{18}$ Esta análise fez perceber que os espectros de RMN ${ }^{1} \mathrm{H}$ e ${ }^{13} \mathrm{C}$ de 3 obtidos em DMSO- $d_{6}$<smiles>[R10]Oc1c([R4])c(O)c([R4])c2oc(-c3ccc(O)c([R3])c3)cc(=O)c12</smiles>

(1) $\mathrm{H}$ Gli $\mathrm{H}$

(2) Gli $\mathrm{H} \quad \mathrm{H}$

(3) $\begin{array}{lll}\mathrm{G} & \mathrm{H} & \mathrm{H}\end{array}$

(4) $\mathrm{H}$ G $\mathrm{H}$

(5) Gli $\mathrm{H} \quad \mathrm{OH}$

(6) $\mathrm{H}$ Gli $\mathrm{OH}$

$\mathbf{G}=\beta-D-G l i-\left(2^{\prime \prime} \rightarrow 1^{\prime \prime \prime}\right)-\alpha-L-R a m$
(7) $\mathbf{R}=\mathbf{R}_{\mathbf{2}}=\mathrm{CH}_{3}, \mathbf{R}_{\mathbf{1}}=\mathbf{H}$

(8) $\mathrm{R}=\mathrm{R}_{\mathbf{1}}=\mathrm{CH}_{3}, \mathrm{R}_{2}=\mathrm{H}$

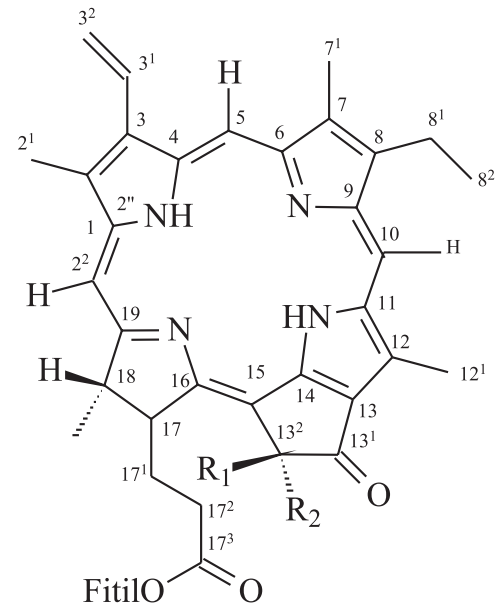<smiles>CCC(/C=C/[C@H](C)C1CCC2C3CC=C4CC(O)CCC4(C)C3CCC21C)C(C)C</smiles>

(9) 22,23-dihidro

(8) $\Delta^{22,23}$<smiles>CCC(C)C1CCC2(C)C(CCC2[C@H](C)/C=C/C(CC)C(C)C)C1</smiles>

(11) $\mathrm{R}_{1}=\mathrm{OH}, \mathrm{R}_{\mathbf{2}}=\mathrm{COOCH}_{3}$

(12) $\mathrm{R}_{1}=\mathrm{COOCH}_{3}, \mathrm{R}_{2}=\mathrm{OH}$

Figura 1. Estrutura das substâncias isoladas de Clusia lanceolata 
exibiram sinais alargados e/ou duplicados, indicando a presença de dois confôrmeros rotaméricos, que foram denominados confôrmeros A e B (Tabela 1; ver material suplementar). No caso de flavonoides, este fenômeno é normalmente observado em 6- $C$-glicosilflavonoides onde a rotação livre no $\mathrm{C}\left(s p^{3}\right)-\mathrm{C}\left(s p^{2}\right)$ da ligação glicose-flavona é impedida por fatores estéricos. ${ }^{18}$ Adicionalmente, os valores não usuais de deslocamento químico observados para H-5"' $\left(\delta_{\mathrm{H}} 2,11\right)$ e H-6" " $\left(\delta_{\mathrm{H}} 0,51\right)$ da unidade de ramnose do dissacarídeo podem estar relacionados a efeitos anisotrópicos do anel aromático da aglicona, decorrentes da conformação adotada por este tipo de substância. ${ }^{20}$ Ainda nos espectros de $\mathrm{RMN}{ }^{1} \mathrm{H}$ e ${ }^{13} \mathrm{C}$ foram observados sinais de menor intensidade, atribuídos a um componente minoritário da mistura, característicos para apigenina substituída na posição C-8 pela mesma unidade de dissacarídeo de 3 . A substância minoritária (4) na mistura foi identificada como vitexina-2"- $O$ - $\alpha$-L-ramnopiranosídeo com base nos sinais adicionais nos espectros de $\mathrm{RMN}{ }^{1} \mathrm{H}$ e ${ }^{13} \mathrm{C}$ e comparação com dados da literatura (Tabela 1). ${ }^{19}$

Tabela 1. Dados (mult. e $J / H z$, em DMSO- $d_{6}$ ) de RMN ${ }^{1} \mathrm{H}(500 \mathrm{MHz})$ e ${ }^{13} \mathrm{C}$ (125 MHz) para os confôrmeros A e B* de (3)e (4)

\begin{tabular}{|c|c|c|c|c|}
\hline \multirow{2}{*}{ Posição } & \multicolumn{2}{|r|}{3} & \multicolumn{2}{|r|}{4} \\
\hline & $\delta_{\mathrm{C}}$ & $\delta_{\mathrm{H}}$ & $\delta_{\mathrm{C}}$ & $\delta_{\mathrm{H}}$ \\
\hline 2 & 163,$5 ; 163,6$ & - & 164,1 & - \\
\hline 3 & 102,$7 ; 102,9$ & $6,77(\mathrm{~s})$ & 102,5 & $6,79(\mathrm{~s})$ \\
\hline 4 & 182,$2 ; 181,9$ & - & 182,2 & - \\
\hline 5 & 161,3 & $13,53(\mathrm{~s}, \mathrm{OH})$ & 160,7 & $13,13(\mathrm{~s}, \mathrm{OH})$ \\
\hline 6 & 109,$0 ; 108,7$ & - & 98,4 & $6,28(\mathrm{~s})$ \\
\hline 7 & 163,4 & - & 162,4 & - \\
\hline 8 & 93,$1 ; 94,3$ & 6,$54 ; 6,52(\mathrm{~s})$ & 104,2 & - \\
\hline 9 & 156,$3 ; 156,5$ & - & 155,9 & - \\
\hline 10 & 104,$3 ; 103,8$ & - & 104,5 & - \\
\hline $1^{\prime}$ & 121,$1 ; 121,2$ & - & 121,6 & - \\
\hline $2^{\prime} ; 6^{\prime}$ & 128,5 & $7,92(\mathrm{~d}, J=8,5)$ & 129,1 & $8,04(\mathrm{~d}, J=8,5)$ \\
\hline $3^{\prime} ; 5^{\prime}$ & 116,1 & $6,93(\mathrm{~d}, J=8,5)$ & 116,0 & $6,91(\mathrm{~d}, J=8,5)$ \\
\hline 4 & 161,3 & - & 161,3 & - \\
\hline $1 "$ & 71,$6 ; 71,4$ & 4,$66 ; 4,62(\mathrm{~d}, J=10,0)$ & 71,5 & $4,76(\mathrm{~d}, J=10,0)$ \\
\hline $2 ”$ & 74,$6 ; 75,8$ & 4,$37 ; 4,18(\mathrm{t}, J=10,0)$ & 75,1 & $4,05(\mathrm{t}, J=10,0)$ \\
\hline $3 "$ & 80,$1 ; 79,7$ & $3,08-3,26(\mathrm{~m})$ & 79,9 & $3,08-3,26(\mathrm{~m})$ \\
\hline 4" & 70,$4 ; 70,5$ & $3,08-3,26(\mathrm{~m})$ & 70,3 & $3,08-3,26(\mathrm{~m})$ \\
\hline $5 "$ & 81,$6 ; 81,5$ & $3,08-3,26(\mathrm{~m})$ & 81,9 & $3,08-3,26(\mathrm{~m})$ \\
\hline $6 "$ & 61,$8 ; 61,3$ & 3,$70 ; 3,76(\mathrm{~d}, J=10,0)$ & 61,2 & $3,70(\mathrm{~d}, J=10,0)$ \\
\hline $1 " ”$ & 100,$4 ; 100,8$ & 5,$08 ; 5,01(\mathrm{sl})$ & 100,4 & 4,98 (sl) \\
\hline $2 "$ & 71,0 & $3,66(\mathrm{~m})$ & 70,5 & $3,60(\mathrm{~m})$ \\
\hline 3 ”" & 70,7 & $3,08-3,18(\mathrm{~m})$ & 70,7 & $3,08-3,18(\mathrm{~m})$ \\
\hline $4 "$, & 71,6 & $2,91(\mathrm{~m})$ & 71,7 & $2,91(\mathrm{~m})$ \\
\hline $5 "$ & 68,3 & $2,29-2,35(\mathrm{~m})$ & 68,3 & $2,29-2,35(\mathrm{~m})$ \\
\hline 6"” & 17,$6 ; 17,9$ & 0,$53 ; 0,61(\mathrm{~d}, J=5,0)$ & 17,8 & $0,47(\mathrm{~d}, J=5,0)$ \\
\hline
\end{tabular}

*Os números em itálico são referentes ao confôrmero B de 3 .

O estudo fitoquímico de espécies do gênero Clusia têm revelado o acúmulo de flavonas $C$-glicosiladas derivadas da apigenina e luteolina, especialmente nas folhas. As substâncias $\mathbf{1}$ e $\mathbf{2}$ foram previamente isoladas em $C$. columnaris e $C$. criuva..$^{21,22}$ A substância $\mathbf{4}$ foi isolada de $C$. sandiensis. ${ }^{23}$ Entretanto, este é o primeiro relato do isolamento da substância $\mathbf{3}$ e das feofitinas $\mathbf{1 1}$ e $\mathbf{1 2}$ na família Clusiaceae. As feofitinas são consideradas produtos de degradação das clorofilas, durante o processo de degradação ocorre a substituição do átomo de $\mathrm{Mg}$ por dois átomos de $\mathrm{H}$, processo conhecido como feofitinização. ${ }^{24}$ Apesar da ocorrência relativamente rara na natureza, a diversidade estrutural das feofitinas e as atividades biológicas que apresentam têm despertado muito interesse.

\section{Atividade antioxidante in vivo dos flavonoides}

Diferentes métodos in vitro e in vivo têm sido desenvolvidos para avaliar a atividade antioxidante de substâncias. Os testes químicos in vitro apesar de serem mais rápidos e simples não são representativos das condições celulares do homem. Neste contexto, os ensaios microbianos in vivo utilizando células eucarióticas da levedura Saccharomyces cerevisiae têm se mostrado adequados, por fornecerem resultados rápidos, reprodutíveis e passíveis de serem correlacionados ao observado no homem..$^{25}$ Neste trabalho, a análise dos resultados foi realizada comparando-se os valores percentuais da viabilidade celular (Figura 2) e disfunção mitocondrial (Tabela 2) da levedura $S$. cerevisiae tratada somente com $\mathrm{H}_{2} \mathrm{O}_{2}(1,0 \mathrm{mM})$ e com este agente estressor após pré-incubação das células com a vitexina (1) e com a mistura contendo isovitexina-2"-O- $-\alpha$-L-ramnopiranosídeo (3) e vitexina-2"-O- $\alpha$-L-ramnopiranosídeo (4), nas concentrações de $25,0 \mu \mathrm{g} \mathrm{mL}^{-1}$ cada. No ensaio de viabilidade celular foram encontradas diferenças estatísticas significativas entre a levedura tratada somente com $\mathrm{H}_{2} \mathrm{O}_{2}$ e tratada com $\mathrm{H}_{2} \mathrm{O}_{2}$ após pré-tratamento com o flavonoide 1 e com a mistura de 3 e 4 . O aumento na viabilidade das células de S. cerevisiae na presença das substâncias sugeriu o efeito protetor dos flavonoides aos danos oxidativos causados pelo agente estressor $\mathrm{H}_{2} \mathrm{O}_{2}$. Além disso, ambas as substâncias também diminuíram ou evitaram os danos causadas à mitocôndria pela solução de peróxido de hidrogênio (1,0 mM) (Tabela 2). Como conclusão dos ensaios, observamos que tanto a substância $\mathbf{1}$ quanto a mistura de $\mathbf{3}$ e $\mathbf{4}$ protegeram as células da ação do peróxido de hidrogênio, mantendo os resultados ao nível experimental do controle. Este é o primeiro relato sobre a avaliação da capacidade antioxidante in vivo das substâncias 1, 3 e 4 utilizando-se como modelo de sistema biológico a levedura $S$. cerevisiae.

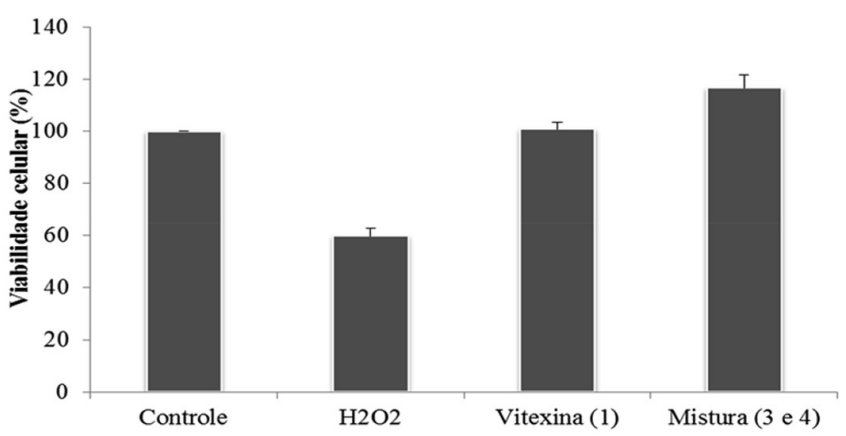

Figura 2. Viabilidade celular da levedura S. cerevisiae nos tratamentos com

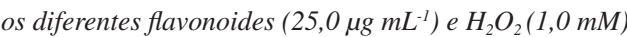

Tabela 2. Dados da disfunção mitocondrial calculado pela diferença entre o número de colônias nos meios YPD 2\% e YPGly 4\%

\begin{tabular}{lc}
\hline Condições & Resultados \\
\hline Controle & 0 \\
$\mathrm{H}_{2} \mathrm{O}_{2}(1,0 \mathrm{mM})$ & $16,9 \pm 3,84$ \\
Vitexina (1) & 0 \\
Mistura (3 e 4) & 0 \\
\hline
\end{tabular}

O peróxido de hidrogênio isoladamente é praticamente inócuo, entretanto pode se difundir facilmente pelas membranas celulares e 
reagir com metais presentes em seu interior e gerar radicais hidroxila. O radical hidroxila causa danos ao DNA, RNA, proteínas, lipídios e membranas celulares do núcleo e mitocondrial. ${ }^{26}$ Alguns flavonoides

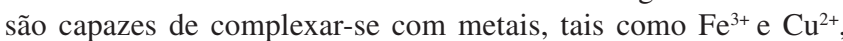
evitando/diminuindo a geração de radicais hidroxila pela reação de Haber-Weiss/Fenton. ${ }^{25}$ Considerando que a geração de radicais hidroxila pelo $\mathrm{H}_{2} \mathrm{O}_{2}$ é mediada por metais de transição, é possível que a propriedade quelante dos flavonoides 1 e da mistura 3 e 4 tenha contribuído para sua atividade antioxidante.

\section{CONCLUSÃO}

Este é o primeiro estudo que relata o isolamento dos metabólitos especiais da espécie $C$. lanceolata e resultou na identificação de 14 substâncias, sendo três inéditas na família Clusiaceae, o flavonoide isovitexina-2"-O- $\alpha-L$-rhamnopiranosídeo (3) e a mistura de feofitinas rel-132-hidroxi-(132-S-)-feofitina a (11), rel-1322-hidroxi-(132-R)feofitina a (12).

Adicionalmente, relatamos a promissora atividade antioxidante in vivo da vitexina (1) e da mistura de isovitexina-2"- $O-\alpha-L$ ramnopiranosídeo (3) e vitexina-2"-O- $\alpha$ - $L$-ramnopiranosídeo (4) empregando-se como modelo de sistema biólogico a levedura $S$. cerevisae. Estes resultados sugerem que $C$. lanceolata é uma nova fonte natural de susbtâncias antioxidantes, que podem vir a atuar futuramente no combate a doenças relacionadas ao estresse oxidativo.

\section{MATERIAL SUPLEMENTAR}

Os espectros de $\mathrm{RMN}$ de ${ }^{1} \mathrm{H}$ e ${ }^{13} \mathrm{C}$ de algumas das substâncias isoladas, citados no texto, estão disponíveis na forma de material suplementar em http://quimicanova.sbq.org.br, em formato PDF, com acesso livre.

\section{AGRADECIMENTOS}

À CAPES, CNPq e a FAPERJ pelas bolsas concedidas e pelo apoio financeiro.

\section{REFERÊNCIAS}

1. Anholeti, M. C.; Paiva, S. R.; Figueiredo, M. R.; Kaplan, M. A. C.; An. Acad. Bras. Ciênc. 2015, 87, 289.

2. Ferreira, R. O.; Carvalho, M. G.; Silva, T. M. S.; Quim. Nova 2012, 35, 2271.

3. Oliveira, C. M. A.; Porto, A. M.; Bittrich, I. V.; Marsaioli, A. J.; Phytochemistry 1999, 50, 1073.
4. Ferreira, R. O.; Silva, T. M. S.; Carvalho, M. G.; Molecules 2015, 20, 14326.

5. Ferreira, R. O.; Camara, C. A.; Agra, M. F.; Silva, T. M. S.; Nat. Prod. Commun. 2012, 7, 1597.

6. Ribeiro, P. R.; Ferraz, C. G.; Guedes, M. L. S.; Martins, D.; Cruz, F. G.; Fitoterapia 2011, 82, 1237.

7. Silva, E. M.; Araújo, R. M.; Freire-Lima, L. G.; Silveira, E. R.; Lopes, N. P.; Paula, J. E.; Braz-Filho, R.; Espindola, L. S.; J. Braz. Chem. Soc. 2013, 24, 1314.

8. Farias, J. A.; Ferro, J. N.; Silva, J. P.; Agra, I. K.; Oliveira, F. M.; Candea, A. L.; Conte, F. P.; Ferraris, F. K.; Henriques, M. ; Conserva, L. M.; Barreto, E.; Inflammation. 2012, 35, 764.

9. Ferreira, R. O.; Carvalho Junior, A. R.; Silva, T. M. G.; Nora, R. N.; Silva, T. M. S.; Carvalho, M. G.; Braz. J. Pharmacog. 2014, 24, 617.

10. Dias, M. M.; Hamerski, L.; Pinto, A.; Quim. Nova 2011, 34, 704.

11. Lima, A. C. A.; Dissertação de Mestrado, Universidade Federal de Alagoas, Brasil, 2000

12. Matsuo, A.; Ono, K.; Nozari, H.; Phytochemistry 1996, 47, 427.

13. Della Greca, M.; Monaco, P.; Previtera, L.; J. Nat. Prod. 1990, 53, 1430.

14. Soares, D. G.; Andreazza, A. C.; Salvador, M.; J. Agric. Food Chem. 2003, 51, 1077.

15. Zhou, X.; Peng, J.; Fan, G.; Wu, Y.; J. Chromatogr. A 2005, 1092, 216.

16. Peng, X.; Zheng, Z.; Cheng, K.-W.; Shan, F.; Ren, G.-X.; Chen, F.; Wang, M.; Food Chem. 2008, 106, 475.

17. Peng, J.; Fan, G.; Hong, Z.; Chai, Y.; Wu, Y.; J. Chromatogr. A 2005, 1074, 111

18. Camargo, L. M. M.; Férézou, J.-P.; Tinoco, L. W.; Kaiser, C. R.; Costa, S. S.; Phytochem. Lett. 2012, 5, 427.

19. Doyama, J. T.; Rodrigues, H. G.; Novelli, E. L. B.; Cereda, E.; Vilegas, W.; J. Ethnopharmacol. 2005, 96, 371.

20. Larionova, M.; Spengler, I.; Nogueiras, C.; Quijanos, L.; RamírezGualito, K.; Cortés-Guzmán, F.; Cuevas, G.; Calderón, J. S.; J. Nat. Prod. 2010, 73, 1623.

21. Compagnone, R. S.; Suarez, A. C.; Leitão, S. G.; Delle Monache, F.; Braz. J. Pharmacog. 2008, 18, 6 .

22. Chedier, L. M.; Paiva, S. R.; Costa, J. L. M.; Figueiredo, M. R.; Kaplan, M. A. C.; J. High Res. Chrom. 1999, 22, 527.

23. Delle Monache, F.; Rev. Latinoam. Quim. 1991, 22, 27.

24. Lanfer-Marquez, U. M.; Rev. Bras. Cienc. Farm. 2003, 39, 227.

25. Soares, D. G.; Andreazza, A. C.; Salvador, M.; Rev. Bras. Cienc. Farm. 2005, 41, 95 .

26. Barreiros, A. L. B. S.; David, J. M.; David, J. P.; Quim. Nova 2006, 29, 113. 